\title{
Interface solutions for interface side effects?
}

\author{
Thomas A. Stoffregen* \\ (*)University of Minnesota, USA \\ E-mail:tas@umn.edu
}

\begin{abstract}
Human-computer interfaces often give rise to a variety of side effects, including eyestrain, headache, fatigue, and motion sickness (aka cybersickness, simulator sickness). We might hope that improvements in interface design would tend to reduce these side effects. Unfortunately, history reveals just the opposite: The incidence and severity of motion sickness (for example) is positively related to the progressive sophistication of display technology and systems.

In this presentation, I enquire about the future of interface technologies in relation to side effects. I review the types of side effects that occur and what is known about the causes of interface side effects. I suggest new ways of understanding relations between interface technologies and side effects, and new ways to approach the problem of interface side effects.
\end{abstract}

\section{Introduction}

Human-computer interfaces have gone through extraordinary development over the past 50 years. Early interfaces consisted of punch cards, followed by lines of text on a screen, followed by graphical user interfaces. Today, development focuses on interfaces that are multimodal (e.g., vision + audition + touch), and which detect and respond to "intuitive" movements of the user.

In a broad sense, interface technology has progressed, or improved. The sophistication, spatiotemporal resolution, and overall quality of interfaces has improved with each passing year. Strangely, steady improvement in interface technologies is associated with a steady increase in reports of side effects among users. Contemporary interfaces can lead to a variety of side effects, including subjective experiences and changes in body movement. Some of these are related to motion sickness, but many are not. For example, head mounted displays can give rise to headache and eyestrain in the absence of motion sickness $[1,2]$.
In addition to subjective aftereffects, virtual environments can bring about changes in body movement, such as visually guided reaching, and the control of standing posture. Changes in movement can be but are not always associated with subjective aftereffects [3]. This is true even for relations between movement aftereffects and motion sickness. For example, prolonged exposure to virtual environments is associated with generalized increases in postural activity [4-7], and with changes in the dynamics of body sway [8, 9] regardless of whether or not participants reported motion sickness. Generalized effects of interface exposure can be independent of changes in bodily movement that precede the onset of motion sickness and occur only among individuals who became motion sick [8, 10]. For example, Dong et al. [11] exposed participants to a high-fidelity console video game, which induced motion sickness in $42 \%$ of participants. Motion sickness was preceded by changes in the variability of head and torso movement, and by changes in the coupling of body movement to the video game. Taken together, these studies show that visual motion can affect multiple parameters of postural activity, and that some of these affects are associated with subsequent motion sickness while others are not. Similar effects occur with non-game virtual environments [9], and in military flight simulators [12].

\section{Good and bad side effects}

Interface side effects can be good. When interfaces simulate real-world systems or situations, then side effects that occur in the real-world setting should also occur in the simulator; this is a form of simulator fidelity or interface realism. As one example, automobile passengers sometimes experience motion sickness, and so motion sickness should sometimes occur among "passengers" in automobile simulations. This form of fidelity applies to vehicle simulation, but it can also apply to aspect of the real world that is reproduced in an interface, such as virtual avatars [13].

This is an Open Access article distributed under the terms of the Creative Commons Attribution-Noncommercial License 3.0, which permits unrestricted use, distribution, and reproduction in any noncommercial medium, provided the original work is properly cited. 
Interface side effects can also be bad. This occurs when side effects occur with interfaces in situations where side effects would not occur in the real world. Drivers rarely experienced motion sickness, but motion sickness is fairly common among "drivers" (i.e., players) of driving video games [11]. Similarly, people do not become motion sick when walking down the street, but motion sickness is common when we control the locomotion of a virtual avatar [13]. Millions of people report motion sickness while playing "first person shooter" games in which they control the locomotion of a character, rather than a vehicle.

In general, motion sickness associated with interfaces that display motion of the user (i.e., selfmotion, or motion of the viewpoint), whether vehicular or otherwise. However, there has not been any systematic research on this issue; we do not know whether motion sickness may occur with other types of contemporary interface systems. Other side effects, such as headache or eyestrain in the absence of motion sickness, are associated with a wide variety of interface systems but, again, there has been no systematic research into relations between the nature of interfaces and the types of side effects that they induce.

\section{It's getting worse}

Reiterating an earlier point, side effects are not going away spontaneously with the development of new interface technologies and systems. Rather, it is just the other way around. Multimodal interfaces and enactive interfaces are wonderful things, true advances in interface technology. While they may be a qualitative leap forward in many senses, they are not by themselves - solutions to the problem of interface side effects. Enactive interface systems, such as Wii and Kinect, are associated with widespread anecdotal reports of motion sickness.

Ignoring this problem is not likely to make it go away. Similarly, the use of disclaimers (e.g., "use of this product may lead to motion sickness") is a legal rather than a practical solution.

Motion sickness is not limited to visual interfaces. Motion sickness has been reported among users of some auditory interfaces [14], and is common in vehicle simulators that include inertial displacement of the body (i.e., motion-base simulators).

\section{Explanations from behavior}

Rather than relying on brute technological development, a meaningful solution to the problem of interface side effects will need to emerge from a better understanding of the perceptual-motor dynamics of human movement. This is, in part, what enactive interface design and the SKILLS project are all about.

Movement relative to the illuminated environment differs from movement relative to the gravitoinertial environment [15]. In this regard, simulation of selfmotion or viewpoint motion cannot be entirely faithful.

\section{Potential solutions}

Given that completely faithful simulation is not possible, the best alternative may be to design interfaces that are deliberately different from the relevant real-world situations.

Consider, as an example, the control of virtual avatars. Human gait moves the eyes through the environment, but also (simultaneously) moves the eyes up and down - the head bobs slightly due to the scissoring action of the legs in walking. Including the visual consequences of this bobbing in virtual environments can increase the literal realism of interfaces, but often has little to do with the functional goals of the interface and is likely to contribute to motion sickness. A virtual avatar that moves in translation without "gait bobbing" has lower nominal realism but may lead to better outcomes.

As another example, consider the design of augmented reality interfaces that might be used to minimize or reduce seasickness. Adaptation to life at sea requires us to learn to control our bodies relative to motion of the ship. Research suggests that this adaptation may be facilitated by the visible horizon [16]. Seasickness often occurs below decks, that is, in the interior of ships where the horizon is not visible. Below decks, stabilization of the body may be facilitated by interfaces or displays that "show" the horizon; one option might be an artificial horizon that is projected onto the walls of cabins.

Deliberate reductions in nominal fidelity might have an additional application. As mentioned earlier (section 2), side effects can be important aspects of interface fidelity, as when passengers in virtual vehicles experience motion sickness that would be experienced by passengers in the corresponding physical vehicle. Deliberate interface infidelity might be used to reduce the risk of side effects in an interface, such that the interface actually had fewer (or lesser) side effects than the corresponding physical system.

\section{Deliberate design}

Interface side effects are a serious problem that impacts the acceptance and functional utility of 
interfaces. It is unlikely that the problem of inappropriate side effects will fade away spontaneously. It is more likely that the problem will be minimized only if researchers and designers devote deliberate, explicit attention to the problem. Effective solutions to the problem of interface side effects are likely to come from understanding of behavior (i.e., perception and action) and the application of such understanding to interface design issues, rather than from developments in interface technologies, as such.

\section{References}

[1] M. H. Draper, E. S. Viirre, V. J. Gawron, and T. A. Furness. The effects of virtual image scale and system delay on simulator sickness within head-coupled virtual environments. Human Factors, 43, 129-146, 2001.

[2] O. Merhi, E. Faugloire, M. Flanagan, and T. A. Stoffregen. Motion sickness, console video games, and head mounted displays. Human Factors, 49, 920-934, 2007.

[3] K. Stanney, G. Salvendy, J. Deisinger, P. DiZio, S. Ellis, J. Ellision, G. Fogleman, J. Gallimore, L. Hettinger, R. Kennedy, J. Lackner, B. Lawson, J. Maida, A. Mead, M. Mon-Williams, D. Newman, T. Piantanida, L. Reeves, O. Riedel, M. Singer, T. Stoffregen, J. Wann, R. Welch, J. Wilson, J., and R. Witmer. Aftereffects and sense of presence in virtual environments: Formulation of a research and development agenda. Report sponsored by the Life Sciences Division at NASA Headquarters. International Journal of Human-Computer Interaction, 10, 135-187, 1998.

[4] H. Akiduki, S. Nishiike, H. Watanabe, K. Matsuoka, T. Kubo, and N. Takeda. Visual-vestibular conflict induced by virtual reality in humans. Neuroscience Letters, 340, 197-200, 2005.

[5] R. S. Kennedy, K. S. Berbaum, and M. G. Lilienthal. Disorientation and postural ataxia following flight simulation. Aviation, Space, and Environmental Medicine, 68, 13-17, 1997.

[6] R. S. Kennedy, J. E. Fowlkes, and M. G. Lilienthal. Postural and performance changes following exposure to flight simulators. Aviation, Space, and Environmental Medicine, 64, 912-920, 1993.
[7] R. S. Kennedy, and K. M. Stanney. Postural instability induced by virtual reality exposure: Development of a certification protocol. International Journal of HumanComputer Interaction, 8, 25-47, 1996.

[8] C. T. Bonnet, E. M. Faugloire, M. A. Riley, B. G. Bardy, and T. A. Stoffregen. Motion sickness preceded by unstable displacements of the center of pressure. Human Movement Science, 25, 800-820, 2006.

[9] S. Villard, M. B. Flanagan, G. Albanese, and T. A. Stoffregen. Postural instability and motion sickness in a virtual moving room. Human Factors, 50, 332-345, 2008.

[10] T. A. Stoffregen and L. J. Smart. Postural instability precedes motion sickness. Brain Research Bulletin, 47, 437-448, 1998.

[11] X. Dong, K. Yoshida, and T. A. Stoffregen. Control of a virtual vehicle influences postural activity and motion sickness. Journal of Experimental Psychology: Applied, in press, 2011.

[12] T. A. Stoffregen, L. J. Hettinger, M. W. Haas, M. Roe, and L. J. Smart. Postural instability and motion sickness in a fixed-base flight simulator. Human Factors, 42, 458-469, 2000.

[13] Y. C. Chen, X. Dong, J. Hagstrom, and T. A. Stoffregen. Control of a video game avatar influences motion sickness and postural activity. Progress in Motor Control VIII, Cincinnati, USA, July 2011.

[14] C. Dicke, V. Aaltonen, and M. Billinghurst. Simulator sickness in mobile spatial sound spaces. Lecture Notes in Computer Science, SpringerLink, Berlin, 287-305, 2010.

[15] T. A. Stoffregen, B. G. Bardy, L. J. Smart, and R. J. Pagulayan. On the nature and evaluation of fidelity in virtual environments. In L. J. Hettinger and M. W. Haas (Eds.), Virtual and adaptive environments: Applications, Implications, and Human Performance Issues (pp. 111128). Mahwah, NJ: Lawrence Erlbaum Associates, Inc., 2003.

[16] A. M. Mayo, M. G. Wade, and T. A. Stoffregen. Postural effects of the horizon on land and at sea. Psychological Science, 22, 118-124, 2011 